Anuario da Facultade de Dereito da Universidade da Coruña

Vol. 22 (2018), pp. 499-510

ISSNe: 2530-6324 || ISSN: 1138-039X

DOI: https://doi.org/10.17979/afdudc.2018.22.0.5201

\title{
LA CONDICIÓN RESOLUTORIA EN LOS ACTOS JURÍDICOS*
}

\author{
ELEONORA NICOSIA \\ Investigadora en el Dipartimento di Giurisprudenza \\ Università di Catania
}

Resumen: La autora trata de identificar en qué tipos de actos podía encontrar aplicación la condición resolutoria en la Antigua Roma.

Palabras clave: ad condicionem; sub condicione; emptio pura

Abstract: The aim of this paper is to identify wich types of acts are the application of a clause providing a resolutive condition in Ancient Rome.

Key words: ad condicionem; sub condicione; emptio pura

SUMARIO: I. BREVE PANORÁMICA DE LAS OPINIONES EXPRESADAS POR LA DOCTRINA.- II. ANÁLISIS DE LAS FUENTES QUE PROPORCIONAN TESTIMONIO DE LA UTILIZACIÓN DE LA CONDICIÓN RESOLUTORIA.- III. OBSERVACIONES FINALES.

* Texto correspondiente a la Ponencia impartida con motivo de la muy amable y agradable invitación que me fue formulada por la Prfra. Margarita Fuenteseca y el Prof. Ramón Rodríguez Montero para participar e intervenir en el Seminario interdisciplinar internacional "El negotium contractum: aspectos históricos y perspectivas de futuro", celebrado respectivamente en las Universidades de Vigo y A Coruña los días 9 y 10 de noviembre de 2017. Asimismo quiero agradecer la revisión de la traducción al idioma español realizada por mi amigo Ramón. 


\section{BREVE PANORÁMICA DE LAS OPINIONES EXPRESADAS POR LA DOCTRINA}

Tres diferentes modalidades accesorias de la manifestación de voluntad podían incorporarse a los actos jurídicos ${ }^{1}$ : el término (dies), la condición (condicio) y, sólo en ciertos tipos de actos, el modus.

Mediante la condición se tenía la posibilidad de hacer depender los efectos del acto al que se incorporaba de un evento futuro y objetivamente incierto. Distinguimos la condición suspensiva, mediante la que la producción de los efectos del acto dependen del cumplimiento del evento futuro e incierto establecido mediante dicha condición, de la condición resolutoria, con la que, por contra, los efectos del acto jurídico cesarán al producirse el evento señalado en la condición.

Desde siempre se ha venido dedicando una gran atención a la condición resolutoria. Precisamente por ello parece oportuno hacer una breve síntesis de las posiciones sostenidas respecto a la misma por la doctrina durante el transcurso del tiempo $^{2}$.

Durante mucho tiempo, tanto en los estudios específicos sobre la condición, como en los Manuales de instituciones de derecho romano se ha enseñado que los juristas romanos habían plena y cabalmente elaborado solo la condición suspensiva. A este único tipo de condición se haría referencia en las fuentes en que se habla de condicio. A la misma habrían dedicado su atención los juristas, preocupándose de analizar las consecuencias que por su incorporación se producían en los diferentes actos, hablando a propósito de negocios sub condicione. Y, dado que no elaboraron el concepto de condición resolutoria, en las hipótesis que deseaban hacer cesar los efectos de un acto cuando se produjese un evento futuro e incierto, construían el negocio como purus, adosando a este negocio un pacto sometido a condición suspensiva, mediante el que se pretendía la resolución del acto principal; si el evento establecido en la condición se daba, el pacto de resolución producía su resultado y provocaba la cesación de los efectos del acto principal al que se añadía. Este mecanismo resolutorio se encuentra ampliamente testimoniado -de forma particular en el ámbito de la compraventa- y descrito con la expresión emptio pura quae sub condicione resolvitur ${ }^{3}$.

\footnotetext{
${ }^{1}$ La extrañeza al planteamiento de los juristas romanos de la configuración de estas tres cláusulas como elementos 'accidentales' del negocio, diferenciados de los elementos 'esenciales', resulta puesta de manifiesta por G. NICOSIA, Profili istituzionali di diritto privato romano (Catania 2017) 207, donde estas cláusulas vienen denominadas modalidades accesorias de la manifestación de voluntad.

${ }^{2}$ Para una relación detallada de la bibliografía, a la que aquí se hace solamente una sintetética alusión, me permito di remitir a mi In diem addictio e lex commissoria (Catania 2013) 1 ss.

${ }^{3}$ Baste recordar aquí las claras palabras de Ulpiano en D. 18.2.2 pr. (Ulp. 28 ad Sab.): Quotiens fundus in diem addicitur, utrum pura emptio est, sed sub condicione resolvitur, an vero condicionalis sit magis emptio, quaestionis est. Et mihi videtur verius interesse, quid actum sit: nam si quidem hoc actum est, ut meliore allata condicione discedatur, erit pura emptio, quae sub
} 
Sin embargo, esta idea, consolidada en la doctrina, fue sometida a crítica en la pimera mitad del siglo pasado por parte de la romanística alemana ${ }^{4}$, y dicha crítica ha enontrado aceptación tanto por algunos sectores de la mencionada romanística alemana como italiana.

En este sentido, se ha considerado que los juristas romanos, habiendo elaborado conceptualmente el concepto que nosotros tenemos de condición resolutoria, no obstante, habrían tenido dificultades para definir este nuevo tipo de condición, por lo que, más que definirla con un adjetivo, a imágen y semejanza de cuanto hicieron con la condición suspensiva (hablando de negocio condicionalis o sub condicione), prefirieron utilizar una perífrasis. Por consiguiente, la expresión emptio pura quae sub condicione resolvitur habría indicado una verdadera y propia condición resolutoria y no el pacto de resolución suspensivamente condicionado. En esta expresión el adjetivo pura habría indicado que el negocio, en este caso la compraventa, se había concluido sin la introducción de una condición suspensiva; sin embargo, esta compraventa se habría resuelto bajo condición (sub condicione resolvitur). Por consiguiente, también en este caso se habría tratado no de dos actos (el negocio principal y el pacto de resolución), sino de un único acto (así como en el negocio suspensivamente condicionado). Y esta condición resolutoria habría encontrado aplicación en el ámbito de los negocios bonae fidei.

Hace casi cuarenta años, un romanista italiano, Albanese ${ }^{5}$, ponía de manifiesto que no solo los romanos habían elaborado perfectamente el concepto de condición resolutoria, sino que también habían acuñado una terminología idónea para indicar este distinto tipo de condición; estos hablaban de negocios ad condicionem en referencia a los tipos en que, mediante la inserción de la condición resolutoria, estaba prevista la duración de los efectos del negocio solamente hasta ad condicionem.

La doctrina más reciente, o continúa enseñando que los juristas romanos no elaboraron el concepto de condición resolutoria; u opera una especie de síntesis de las varias opiniones, considerando que cuando a un negocio se añadía una condición resolutoria, se hablaba recíprocamente de negocio ad condicionem o de negocio puro, pero que sub condicione resolvitur; o bien, a propósito de la emptio pura quae sub condicione resolvitur, habla directamente de venta con condición resolutoria (o, finalmente, omite el problema del significado de la expresión).

condicone resolvitur: sin autem hoc actum est, ut perficiatur emptio, nisi melior condicio offeratur, erit emptio condicionalis.

${ }^{4}$ R. HENLE, Die rechtliche Natur der in diem addictio beim Kaufvertrage, in Festschrift Koschaker (Weimar 1939) 169 ss., v. 190 s.

${ }^{5}$ Gli atti negoziali nel diritto privato romano (Palermo 1982) 272 ss. 


\section{ANÁLISIS DE LAS FUENTES QUE PROPORCIONAN TESTIMONIO DE LA UTILIZACIÓN DE LA CONDICIÓN RESOLUTORIA}

Creo que para intentar poner orden en la materia resulta oportuno examinar los pasajes en que se encuentra la expresión ad condicionem, y ver cómo funcionaba en concreto, para, a continuación, dilucidar si el efecto resolutivo descrito en las fuentes con la expresión emptio pura quae sub condicione resolvitur se encuentra conectado al funcionamiento de la condición resolutoria.

Antes parece oportuno realizar algunas breves puntualizaciones.

Los pasajes en los que se habla de actos ad condicionem son un número limitado y se encuentran extraídos de obras pertenecientes a varios juristas: Ulpiano, Paulo, Papiniano (también se recogen referencias a la condición resolutoria en Labeon, Javoleno y Trifonino).

Además, resulta preciso subrayar que en la mayor parte de estos pasajes al acto sub condicione se contrapone el acto ad condicionem. Existe, por tanto, una clara consciencia en los juristas respecto a la identificación de la condición y del diferente funcionamiento sobre los efectos del acto que la misma produce, según sea suspensiva o resolutoria.

Veamos esos testimonios, comenzando por:

D. 26.1.14.5 (Ulp. 37 ad Sab.): Sed et si ad certam condicionem datus sit, aeque evenit ut desinat esse tutor exsistente condicione.

En la parte del fragmento que precede a este párrafo, Ulpiano se ocupaba de otras hipótesis de cesación en la tutela ${ }^{6}$ (del mismo modo que en este pasaje: aeque evenit).

Ulpiano se está ocupando de la hipótesis en que el nombramiento del tutor haya sido dispuesto ad condicionem por el testador. El adjetivo certa que acompaña al sustantivo condicio tiene el significado de determinada: el jurista quiere decir que el tutor es tal hasta cumplirse el evento específico que ha sido establecido en la condición; siendo obviamente incierto si tal evento acaecerá o no. En la hipótesis en que se cumpliese la condicio, el tutor dejará de serlo (ut desinat esse tutor). La terminología utilizada por Ulpiano es la técnica que aparece también en materia de condición suspensiva. También en caso de condición resolutoria los efectos del acto se modificarán existente condicione, es decir, al producirse el evento señalado en la condición. Y, por consiguiente, el tutor dejará de ser tal en el momento en que la condición se cumpla: la condición actúa de forma automática (e inmediata) sobre los efectos del acto.

${ }^{6}$ D. 26.1.14.3 (Ulp. 37 ad Sab.): Sed et si ad tempus fuerit quis datus, tempore finito tutor esse desinit. 4. Praeterea si suspectus quis fuerit remotus, desinit esse tutor. 
Asimismo, en materia de datio tutoris, debemos de tomar en consideración otros dos pasajes. Ante todo:

D. 26.2.8.2 (Ulp. 24 ad Sab.): Tutorem autem et a certo tempore dare et usque ad certum tempus licet et sub condicione et usque ad condicionem.

Ulpiano contrapone a la hipótesis del tutor a tempore datus, la del tutor dado ad tempus, y sucesivamente contrapone la hipótesis del tutor dado sub condicione a la hipótesis del tutor ad condicionem datus. Por tanto, el jurista no solo contrapone la condición resolutoria a la condición suspensiva, recurriendo a una utilización consciente de la teminología técnica, sino que, además, inserta esta distinción en una visión más amplia (que se encuentra presente en algunos de los otros pasajes que a continuación examinaremos) que comprende también el término; más exactamente, los dos tipos de término, inicial y final, abstractamente aplicables a los negocios. Así:

D. 26.10.3.7 (Ulp. 35 ad ed.): ... si proponas aliquem desisse esse tutorem et rursum coepisse (ut puta usque ad tempus vel ad condicionem erat datus, deinde iterum vel superveniente condicione testamentaria vel etiam a praetore datus est) ...

También en este texto, Ulpiano no solo distingue los dos distintos tipos de condición, recurriendo aquí asimismo a la terminología técnica específica, ad condicionem, sino que, una vez más, hace referencia también al término.

Veamos con más detenimiento el pasaje: Ulpiano plantea la hipótesis del que deja de ser tutor para, posteriormente, volver a serlo; $y$, en primer lugar, pone el ejemplo del tutor ad condicionem datus, que deja de de ser tal (desisse esse tutorem), obviamente al cumplirse la condición, y que sucesivamente vuelve a ser tutor (rursum coepisse) superveniente condicione, esto es, cuando se cumple una condición suspensiva a la que estaba subordinado su nombramiento. Por tanto, Ulpiano distingue con claridad y conscientemente la condición resolutoria de la condición suspensiva. Y al cumplimiento de la condición resolutoria el efecto automático que sigue es que el tutor deja de ser tal (desisse esse tutorem). Pero no sólo esto: Ulpiano aproxima, si bien con cautela, el término final a la condición resolutoria. En efecto, no hay duda alguna de que la finalización de la carga de tutor puede ser desplazada a un momento sucesivo y que tal cese pueda ser ligado a un evento cierto (vencimiento del término), o a un evento incierto (eventual producción del acontecimiento establecido en la condición resolutoria); en todo caso, el efecto será siempre el mismo: el tutor dejará de ser tal.

Una concreta ejemplifcación de una condición resolutoria añadida al nombramiento de un tutor se encuentra en un pasaje de Trifonino:

D. 27.1.45.1 (Tryphon. 13 disput.): Sed si ita scriptum in testamento fuit: 'Titius tutor esto: cum rei publicae causa aberit, tutor ne esto: cum redierit, tutor esto'... 
También Trifonino proporciona el ejemplo de una doble datio tutoris establecida para la misma persona. Para la primera datio se introduce una condicón resolutoria y para la segunda una condición suspensiva: en el primer caso, la terminación de la tutela depende de un evento incierto, la ausencia rei publicae causa; en el segundo, la recuperación de la carga de tutor depende de otro evento igualmente incierto, el retorno (la finalización de la función rei publicae causa).

En este pasaje se puede ver claramente cómo al producirse el evento establecido en la condición resolutoria -la ausencia rei publicae causa-, el tutor nombrado cesa inmediatamente en su carga. También, por otra parte, cómo al producirse el evento establecido en la condición suspensiva -la finalizacion de la carga públicaautomáticamente vuelve a ser tutor.

A la condición resolutoria en una disposición de última voluntad también se hace referencia en:

D. 29.1.15.4 (Ulp. 45 ad ed.): Miles et ad tempus heredem facere potest et alium post tempus vel ex condicione vel in condicionem.

Las disposiciones contenidas en el testamento militar gozaban de un tratamiento especial, dado que se consideraban válidas también en las hipotesis en que siguiendo las reglas normales no habrían debido serlo. Como explica Ulpiano, al miles se le permitía introducir un término final y un término inicial a la institutio heredis, o también una condición suspensiva y una condición resolutoria. Al militar se le permitía disponer que alguien fuese su heredero hasta un cierto momento, y que otro lo fuese a paritir de otro momento; o, igualmente, que alguien fuese heredero hasta cumplirse el evento establecido en la condicion (resolutoria), y que otro fuese heredero a partir del cumplimiento del evento establecido en la condición (suspensiva). Es singular que en el pasaje Ulpiano utlice a propósto de la condición las partículas in y ex (para indicar, respectivamente, la condición resolutoria y la suspensiva), que son más recurrentes en relación al término (in diem e ex die). Y también en este pasaje Ulpiano hace una cuádruple exposición de los dos tipos de términos y de los dos tipos de condición, poniendo en paralelo el término final y la condición resolutoria, y el término inicial y la condición suspensiva.

La expresión ad condicionem aparece también en un ámbito distinto al de las disposiciones de última voluntad, donde, como se ha visto, respecto de algunas disposiciones, referidas en particular a la datio tutoris, no existían dudas de que pudiesen ser establecidas ad condicionem. Por contra, en cuanto a otros actos jurídicos, los juristas se plantearon el problema de establecer si aquellos pudiesen ser concluidos ad condicionem, es decir, si pudiese encontrar aplicación en los mismos la condición resolutoria.

Veamos, sobre todo, un pasaje de Papiniano: 
D. 8.1.4 pr. (Pap. 7 quaest.): Servitutes ipso quidem iure neque ex tempore neque ad tempus neque sub condicione neque ad certam condicionem... constitui possunt...

Papiniano excluye que se pueda constituir una servidumbre no solo ad condicionem (también aquí, como en el pasaje de Ulpiano, el adjetivo certa, que acompaña al sustantivo condicio, tiene el significado de determinada: el jurista se plantea la duda de si el derecho de servidumbre se puede extinguir al verificarse el evento que se contiene en la condición; siendo obviamente incierto si tal evento se producirá o no), sino, más en general, con cualquier cláusula que pueda limitar su duración. No se puede, por tanto, establecer en una constitución de sevidumbre ni un término final, ni una condición suspensiva, ni una condición resolutoria. También se presenta en Papiniano la terminología técnica específica idónea para indicar tanto los dos tipos de término como los dos tipos de condición; refiriéndose a la condición resolutoria, el jurista habla, en efecto, de servitus ad condicionem, en contraposición a la servitus sub condicione. Por tanto, esto demuestra que los juristas romanos no sólo habian elaborado plenamente la condición resolutoria, que contraponían a la suspensiva, sino también pone de manifiesto que crearon una terminología técnica idónea a la que recurrían cuando debían hablar de este tipo de condición, con el que se preveía que los efectos del acto durasen ad condicionem.

Ademas, también Papiniano hace una cuádruple exposición de las cláusulas abstractamente aplicables a un acto jurídico, esto es, los dos tipos de término y los dos tipos de condición. Y en este elenco coloca primero las cláusulas que desplazan a un momento sucesivo la producción de los efectos del acto (término inicial y condición suspensiva) y después las cláusulas que, por el contrario, preveen la inmediata producción de los efectos del acto y su finalización en un momento posterior (término final y condición resolutoria).

A continuación:

Vat. Fr. 50 (Paul. 1 man.): In mancipatione vel in iure cessione an deduci possit vel ex tempore vel ad tempus vel ex condicione vel ad condicionem, dubium est...

A propósito de la deductio ususfructus en una mancipatio o en una in iure cessio, Paulo expone como dudosa la posibilidad de que se puedan añadir tanto los dos tipos de término como los de condición. También en este pasaje encontramos, por tanto, la cuádruple formulación de las cláusulas que potencialmente se podían agregar a un acto jurídico. Asimismo, en el citado pasaje, dichas cláusulas aparecen colocadas de forma paralela: en la primera de las dos contraposiciones, antes la cláusula que pospone a un momento sucesivo la producción de los efectos del acto (término inicial y condición susensiva); y después la cláusula que, por el contrario, prevee la inmediata producción de los efectos del acto y su finalización en un momento sucesivo (término final y condición resolutoria).

También Paulo utiliza de forma consciente la terminología técnica idónea para 
indicar la condición resolutoria (ad condicionem) en contraposición a la terminología técnica utilizada para indicar la condición suspensiva; al respecto se debe advertir cómo Paulo utilizó la partícula ex, seguramente menos usual, pero recurrente en las fuentes (ya la encontramos en D. 29.1.15.4, de Ulpiano), para introducir la condición suspensiva (antes que la más difundida partícula $s u b$ ).

Un ejemplo de utilización concreta de la condición resolutoria en una constitución de usufructo parece que se puede extraer de:

D. 33.2 .30 pr. (Iavol. 2 ex poster. Lab.): Cui usus fructus legatus esset, donec ei totius dotis satisfieret, cum ei heres pro sua parte satis dedisset, quamvis reliqui satis non darent, tamen pro ea parte usum fructum desinere habere mulierem ait Labeo...

Javoleno refiere la opinión expresada por Labeón a propósito de un legado de usufructo dispuesto por el marido a favor de la mujer. El derecho de usufructo de la mujer se extinguiría en el momento en que se prestase garantía (satisfactio) por el importe total de la dote. La hipótesis expuesta al jurista debió ser aquella en que existiesen varios herederos: en efecto, se habla de satisfactio prestada pro parte por el heredero. Y también en este caso el usufructo se habría extinguido pro ea parte, es decir, en medida proporcional a la parte de dote por la que satis dedisset. No cabe duda de que en este caso se haya aplicado al legado de usufructo una condición resolutoria; la mujer será titular del derecho de usufructo, y tal derecho se extinguirá sólo en el momento en que se preste la satisfactio por el importe completo de la dote, y, en opinión de los juristas, tal derecho se extinguirá también pro parte, en proporción a la parte de satisfactio prestada. La finalización del derecho constituido depende, por consiguiente, de un evento futuro y objetivamente incierto, es decir, de una condición resolutoria.

Finalmente, un ejemplo de utilización concreta de la condición resolutoria en el ámbito de la stipulatio parece poder extraerse de:

D. 44.7.44.2 (Paul. 74 ad ed. praet.): Condicio vero efficax est, quae in constituenda obligatione inseritur, non quae post perfectam eam ponitur, veluti 'centum dare spondes, nisi navis ex Asia venerit?'...

Paulo está explicando que para que la condición sea efficax resulta necesario que la misma se introduzca in constituenda obligatione y no post perfectam eam. Proporciona después el ejemplo de una stipulatio, negocio obligatorio por excelencia, a la que ha sido agregada la condición 'nisi navis ex Asia venerit?'. Esta condición ejemplificada por Paulo, como desde hace tiempo ha puesto de manifiesto la doctrina, es una condición resolutoria ${ }^{7}$.

\footnotetext{
${ }^{7}$ V. mi In diem addictio cit., 11 nt. 23. Sin embargo, se ha discutido por qué según Paulo esta condición por él ejemplificada no es efficax. La doctrina se ha dividido al respecto en dos orientaciones: por una parte, los autores que han considerado que Paulo negase la posibilidad de la introducción de una condición resolutoria a una obligación; por otra, aquellos que han creído que la stipulatio en la que se introduce la condición, tal y como la ejemplifica Paulo, presenta un vicio de forma: el verbo spondeo habría debido cerrar la interrogación estipulatoria, es decir, en el
} 


\section{OBSERVACIONES FINALES}

De las fuentes hasta aquí analizadas resulta indudable que los juristas romanos elaboraron plenamente la condición resolutoria, es decir, que concibieron la posibilidad de que un acto jurídico dejase automáticamente de producir sus efectos en el momento en que el evento establecido en la condición se verificase. Y vimos también cómo las fuentes testimonian para qué actos concretos consideraron utilizable este tipo de condición. Además, los textos nos informan de la utilización de una terminología técnica específica (en contraposición a la terminología utilizada para la condición suspensiva) para indicar este tipo de condición.

En atención a estos datos reflejados en las fuentes examinadas podemos valorar el significado de otra expresión, también recogida en las fuentes, emptio pura quae sub condicione resolvitur, para intentar comprender si también con la misma los juristas se estuviesen refiriendo a la condición resolutoria.

Ciertamente, un primer argumento para creer que la expresión emptio pura quae sub condicione resolvitur no indicase una venta a la que se añadía una condición resolutoria se fundamenta propiamente en el dato terminológico: los juristas habían elaborado una terminología técnica específica, idónea para indicar la condición resolutoria, hablando de negocios ad condicionem. Además, es un dato incontestable que utilizan la expresión emptio pura quae sub condicione resolvitur también aquellos juristas que hablan de negocios ad condicionem, y, en ocasiones, en el interior de la misma obra ${ }^{8}$.

De cualquier forma, existen otros argumentos que demuestran que con la expresión emptio pura quae sub condicione resolvitur los juristas no indicaban una venta con condición resolutoria. El sector de la doctrina que ha querido identificar la condición resolutoria en esta expresión, ha afirmado para defender esta idea que en esta expresión el adjetivo pura significaba que la compraventa había sido concluída sin la inclusión de una condición suspensiva. Pero esta presuposición ciertamente es errónea. En efecto, se encuentra ampliamente testimoniado en las fuentes que con el adjetivo purus (y el adverbio pure) se indicaban los actos concluidos sin inclusión de término o condición para contraponerlos a estos. Solo a título ejemplificativo se pueden citar, sobre todo: Gayo (G. 1.186: Et ideo si cui testamento tutor sub condicione aut ex die certo datus sit, quamdiu condicio aut dies pendet, tutor dari potest; item si pure datus fuerit, quamdiu nemo heres existat); Marciano en D. 20.1 .5 pr. (et sive pura est obligatio vel in diem vel sub condicione); Florentino en D. 45.2 .7 (nec enim

ejemplo presentado por Paulo, el error residiría en haber pronunciado las palabras nisi navis ex Asia venerit? tras pronunciar el verbo spondere.

${ }^{8}$ Así Ulpiano en los libri ad Sabinum de los que se extraen tanto D. 18.2.2 pr. (donde encontramos la expresión pura emptio est, sed sub condicione resolvitur), cuanto D. 26.1.14.5 e D. 26.2.8.2 (en que figura la expresión ad condicionem). 
impedimento erit dies aut condicio, quo minus ab eo, qui pure obligatus est, petatur); y, en más lugares, Ulpiano: en D. 28.5.9.19 (Sed et si pure fuerit heres institutus libertate in diem data), en D. 46.1.6.1 (Et parvi refert, utrum pure fideiussorem obligem an ex die an sub condicione), en D. 46.1.27 pr. (Si plures sint fideiussores, unus pure, alius in diem vel sub condicione acceptus), en D. 50.16.10 ('Creditores' accipiendos esse constat eos, quibus debetur... sive pure sive in diem vel sub condicione).

Pero el argumento decisivo es otro: hay que tener presente como cesaban concretamente los efectos de esta emptio pura quae sub condicione resolvitur. Esta expresión se utilizaba por los juristas para describir el efecto que sobre la venta tenían dos cláusulas que frecuentemente encontraban aplicación en la compraventa in diem addictio e lex commissoria. Ambas se establecían a favor del vendedor: con la primera, éste se reservaba la posibilidad de rescindir la primera compraventa para concluir una segunda compraventa con un mejor oferente; con la segunda, el vendedor se reservba la posibilidad de rescindir la compraventa cuando el comprador no pagase el precio en la fecha acordada. Más, no obstante, los juristas afirman que las suertes de la venta con in diem addictio dependen de la presentación de una mejor oferta (si melior condicio allata sit), y que las suertes de la venta con lex commissoria dependen del pago del precio (si pecunia soluta non sit); sin embargo, cuando explican en esencia el funcionamiento de estas cláusulas, no dejan de subrayar puntualmente, de vez en cuando, cómo el cumplimiento de la condición sea el presupuesto para que el vendedor pueda ejercitar las facultades previstas con las dos cláusulas: las suertes de la venta dependen de la voluntad del vendedor; a éste se remite la posiblidad de rescindir la venta sólo después y siempre que una melior condicio le haya sido propuesta, o que el precio no se le haya pagado. Y es éste papel desarrollado por la voluntad del vendedor en orden a las suertes de la venta el que me parece que generalmente no se ha tomado debidamente en cuenta. Los juristas no sustentan dudas sobre el hecho de que, en una venta con in diem addictio, el vendedor también pueda no aceptar la mejor oferta sucesiva y optar por continuar haciendo valer la primera venta, renunciando a concluir la segunda, aunque objetivamente resulte más ventajosa. Así mismo, creen indudable que el vendedor, en una venta con lex commissoria, cuando no haya sido pagado el precio, pueda actuar para obtener el cumplimiento por parte del comprador, antes que rescindir la venta.

Todo lo indicado resulta probado por los textos que se ocupan de estas cláusuas. A título ejemplificativo es suficiente observar dos pasajes en tema de in diem addictio:

D. 18.2 .9 (Ulp. 28 ad Sab.): ... licere venditori meliorem condicionem oblatam abicere sequique primam quasi meliorem...

D. 18.2.14.2 (Paul. 5 ad Sab.): ... ea melior intellegitur quam venditor comprobavit, cui licuit non addicere.

Y dos fragmentos en tema de lex commissoria: 
D. 18.3.3 (Ulp. 30 ad ed.): Nam legem commissoriam, quae in venditionibus adicitur, si volet venditor exercebit, non etiam invitus.

D. 18.3.4.2 (Ulp. 32 ad ed.): ... statim atque commissa lex est statuere venditorem debere, utrum commissoriam velit exercere an potius pretium petere...

Por consiguiente, la resolución de la compraventa no se produce automáticamente, según vimos testimoniado en los pasajes que se ocupan del funcionamiento de la condición resolutoria: en los mismos la verificación del evento hacía cesar inmediatamente y de manera automática los efectos del acto.

Sin embargo, en el caso de la emptio pura quae sub condicione resolvitur, cuando se produjese el evento (se presenta una oferta mejor, o el precio no se paga), la venta sigue siendo eficaz, a no ser que el vendedor decida que deje de ser eficaz.

Por tanto, la venta es pura, perfecta, e inmediatamente productora de efectos; a ésta se añade un pacto de resolución suspensivamente condicionado. Al cumplirse el evento establecido en la condición suspensiva, corresponderá al vendedor poder decidir si utilizar o no este pacto de resolución planteado a su favor.

El mismo testimonio nos ofrece otro pasaje, el último sobre el que conviene hacer alguna indicación.

D. 18.3.2 (Pomp. 35 ad Sab.): Cum venditor fundi in lege ita caverit: 'si ad diem pecunia soluta non sit, ut fundus inemptus sit', ita accipitur inemptus esse fundus, si venditor inemptum eum esse velit, quia id venditoris causa caveretur: nam si aliter acciperetur, exusta villa in potestate emptoris futurum, ut non dando pecuniam inemptum faceret fundum, qui eius periculo fuisset.

También Pomponio, a propósito de la venta con lex commissoria, explica cómo el acuerdo si ad diem etc., es interpretado en el sentido de que la venta se rescindirá sólo si esa es la voluntad del vendedor, dado que la cláusula está establecida a su favor. Y añade -y esto es lo que más nos interesa- que no pude haber lugar a una interpretación distinta; en efecto, si se interpretase de forma diferente, se dejaría al comprador la posibilidad de decidir (potestas) las suertes de la venta, simplemente no pagando el precio. Si se hubiese tratado de condición resolutoria, la falta de pago del precio habría conllevado la inmediata reslución de la venta. Y es propiamente ésta la consecuencia que Pomponio excluye: aun cuando se produzca la verificación del evento, la venta solamente se resolverá si ésta es la voluntad del vendedor. 


\section{BIBLIOGRAFÍA}

Albanese, B., Gli atti negoziali nel diritto privato romano (Palermo 1982)

HENLE, R., Die rechtliche Natur der in diem addictio beim Kaufvertrage, in Festschrift Koschaker (Weimar 1939) 169 ss.

NiCOSIA, E., In diem addictio e lex commissoria (Catania 2013)

NiCOSIA, G., Profili istituzionali di diritto privato romano (Catania 2017) 